\title{
Personal identification based on skin texture features from the forearm and multi-modal imaging
}

\author{
${\text { Francesco Bianconi }{ }^{\mathrm{a}, \mathrm{c}, *}, \text { Elena Chirikhina }}^{\mathrm{b}, \mathrm{d}}$, Fabrizio Smeraldi $^{\mathrm{c}}$, Christos Bontozoglou $^{\mathrm{b}}$, Perry Xiao $^{\mathrm{b}}$ \\ ${ }^{a}$ Department of Engineering, Università degli Studi di Perugia \\ Via G. Duranti 93, 06125 Perugia, Italy \\ Tel. +39075 5853706, Fax +39075 5853703 \\ bianco@ieee.org \\ ${ }^{b}$ School of Engineering, London South Bank University \\ 103 Borough Road, London SE1 OAA, United Kingdom \\ perry.xiao,bontozoc@lsbu.ac.uk \\ ${ }^{c}$ School of Electrical Engineering and Computer Science, Queen Mary, University of London \\ Mile End Road, London E1 4NS, United Kingdom \\ f.smeraldi@@qmul.ac.uk \\ ${ }^{d}$ Sinlen Beauty Clinic \\ Elm Parade, Main Rd, Sidcup DA14 6NF, United Kingdom \\ info@sinlen.co.uk
}

Background/purpose. We investigate the use of skin texture features from the inner forearm as a means for personal identification. The forearm offers a number of potential advantages in that it is a fairly accessible area, and, compared with other zones such as fingertips, is less exposed to the elements and more shielded from wear.

Methods. We extract and combine skin textural features from two imaging devices (optical and capacitive) with the aim of discriminating between different individuals. Skin texture images from 43 subjects were acquired from three different body parts (back of the hand, forearm and palm); testing used the two sensors either separately or in combination.

Results. Skin texture features from the forearm proved effective for discriminating between different individuals with overall recognition accuracy approaching $96 \%$.

Conclusions. We found that skin texture features from the forearm are highly individual-specific and therefore suitable for personal identification. Interestingly, forearm skin texture features yielded significantly better accuracy compared to the skin of the back of the hand and of the palm of the same subjects.

Keywords. Skin texture - Personal identification - Image processing - Texture analysis

\section{Introduction}

Skin texture is the result of a number of factors related to age and ethnicity [1] - but also individual-specific features, and for this reason has often been used for biometric authentication. Starting from early works on face authentication, for instance Eigenfaces [2], skin texture has played a prominent role as it covers most of the image, though no texture-specific descriptors were used at that stage. Later works based on elastic graph matching [3, 4] or local descriptors [5] used local variations in facial texture to anchor the reference grid or detect salient points. Likewise, newer approaches such as palm vein recognition [6] implicitly involve a certain amount of skin texture

${ }^{*}$ Corresponding author. Performed part of this work as an academic visitor in the School of Electrical Engineering and Computer Science at Queen Mary, University of London. 
information in the classification step. Of late, the use of skin texture for personal identification (besides fingerprints) has been advocated by various authors; however, most work has so far focussed on the skin of the hands or of the face, arguably the most accessible regions. Other relatively easily accessible areas of the skin such as the forearm have been largely ignored so far. Yet the use of skin texture features from the forearm has a number of potential advantages in that the area is fairly easily accessible, and, compared with other zones such as fingertips, less exposed to the elements and more shielded from wear.

Herein we propose the use of skin texture features from the forearm as a means for personal identification. To this end we developed a prototype biometric system fed with skin images from two different imaging devices: a standard optical dermoscope and an innovative capacitive sensor. Using a database of 1032 skin texture images from 43 different subjects we show that reliable recognition can be achieved with accuracy in excess of $95 \%$ using multiscale texture descriptors in combination with a support vector machine (SVM) classifier.

In the remainder of the paper we provide a description of the materials (Sec. 2) and methods (Sec. 3) used in this study. The experimental set-up and the results are detailed in Secs. 4-5, followed by concluding remarks (Sec. 6), limitations and possible directions for future studies (Sec. 7).

\section{Materials}

A total of 1032 images from 43 subjects ( 24 males, 19 females), average age $32.1 \pm 14.2$ were considered in this study. The ethnic composition [7] of the participants was: White (27), Asian / Asian British (7), Black / African / Caribbean / Black British (7) and Mixed / multiple ethnic groups (2). Skin image acquisition was carried out using two different systems: a capacity fingerprint device (Epsilon, Biox Systems Ltd, United Kingdom) and a digital optic dermoscope (ProScope HR2, Bodelin Technologies, United States). Both devices are described in detail in Secs. 2.12.2. Four images were acquired in both modes from the back of the hand, forearm and palm of the upper left limb of each of the participants, resulting in a dataset of $43 \times 4 \times 3 \times 2=1032$ images (snapshots of the acquisition procedure are shown in Fig. 3). Within each zone the images were taken in different areas and with no predefined relative position nor orientation between the sensors and the inspected surface. Sample images acquired with both systems are shown in Figs. 1-2.

Figure 1: Samples of capacitive images acquired with Epsilon. The approximate scale (length on paper / length on inspected area) is 1:1.

Figure 2: Samples of dermoscopic images acquired with Proscope HR2. The approximate scale (length on paper / length on inspected area) is 3:1.

Figure 3: Image acquisition: Epsilon (left) and Proscope (right).

\subsection{Epsilon}

Epsilon is a capacitive imaging device developed at London South Bank University [8] featuring an active matrix of $256 \mathrm{px} \times 300 \mathrm{px}$, with50 $\mu \mathrm{m}$ spatial resolution per pixel and an inspection area of $12.8 \mathrm{~mm} \times 15 \mathrm{~mm}$. The device generates a capacitance map of the skin surface, which correlates with the content of water in the skin [9]. In our experiments, in order to avoid edge effects, the resulting images were cropped to central portion of dimension $218 \mathrm{px}$ $\times 256 \mathrm{px}$.

\subsection{Proscope}

The Proscope HR2 $2^{\mathrm{TM}}$ is a digital microscope/dermoscope equipped with LED lighting and a 2.0 megapixel sensor, that can capture images up to a resolution of $1600 \mathrm{px} \times 1200 \mathrm{px}$ [10]. In this study the device was fitted with a cross polarized $30 \times$ lens providing a spatial resolution of $\approx 9 \mu \mathrm{m}$ per pixel. The resulting colour images were converted to gray-scale; no spatial crop was applied in this case. 


\section{Methods}

A prototype system for multi-modal personal identification was developed based on the three blocks desrcibed below, i.e.: 1) feature extraction, 2) feature combination and 3) classification.

\subsection{Feature extraction}

Feature extraction was based on three classes of texture descriptors: Gabor filters, Grey-level Co-occurrence Matrices (GLCM) and Local Binary Patterns (LBP). Since all the images were taken at a fixed distance from the inspected area with both systems no scale adjustment was required. However, we used in all cases rotation-invariant features since the orientation of the acquisition devices was unconstrained (see Sec. 2). Details about the parameters of each texture descriptor along with a synoptic table (Tab. 1) are given below.

\subsubsection{Gabor filters}

We used a bank of 24 filters (four frequencies and six orientations). The maximum frequency $\left(F_{M}\right)$ was set to $0.327 \mathrm{px}^{-1}$ and the lower frequencies obtained through octave spacing from $F_{M}$. The smoothing parameters $\eta$ and $\gamma$ were set to $=1.1$ and 0.8 , respectively [11]. The image features were the mean and standard deviation of the absolute value of each transformed image, giving a total of $24 \times 2=48$ features. Rotation-invariant features were obtained by taking, for each frequency, the absolute value of the discrete Fourier transform (DFT) over the six orientations[12]. In the remainder we indicate this set of features as Gabor $_{4,6}^{r i}$.

\subsubsection{Co-occurrence matrices}

We considered co-occurrence matrices [13] generated by sense-symmetric displacement vectors defining digital circles of radii $R=1$ and 2 as described in Fig. 4 .

Figure 4: Neighbourhood arrangement for GLCM features.

From each matrix we extracted the following five parameters: contrast, correlation, energy, entropy and homogeneity [14]. These settings produced $4 \times 5=20$ features for $R=1$ and $6 \times 5=30$ features for $R=2$, for a total of 50 features. Sensitivity to image rotation was removed by taking, for each value of $R$, the absolute value of the DFT of each group of features [14]. In the following section these features will be indicated as $\mathrm{GLCM}_{R}^{r i}$

\subsubsection{Local Binary Patterns}

Local Binary Pattern (LBP) histograms consider the occurrence probability of the binary patterns that arise from a group of points arranged on a circle when thresholded at the value of the central point. LBP operators are by convention labelled with subscript $(m, R)$ indicating the radius $(R)$ and the number of perypheral points $(m)$, and a superscript representing one of the possible variants of the method - see Ref. [15] for details. Herein we used rotation-invariant uniform LBP generated by two concentric digital circles of radii $R=1 \mathrm{px}$ and $R=2 \mathrm{px}$ (Fig. 5). The concatenation of the two variants, which in the remainder we indicate as $\mathrm{LBP}_{8,1}^{\text {riu } 2}+\mathrm{LBP}_{16,2}^{\text {riu } 2}$, generates $10+18=28$ features.

Figure 5: Neighbourhood arrangement for LBP.

\subsection{Feature normalization and combination}

Combination of information from multiple sources was performed through feature concatenation (also referred to as early fusion). Any feature vector resulting from the descriptors introduced in Secs. 3.1.1-3.1.3, e.g. Gabor ${ }_{4,6}^{r i}$, $\mathrm{GLCM}_{1}^{r i}, \mathrm{LBP}_{8,11}^{r i u 2}$, is separately normalised to sum one; concatenation between features is indicated with the symbol '十'. 
Figure 6: Overall accuracy by image descriptor, image source, acquisition zone and classifier: scatter plots

Figure 7: Accuracy by combination of features/image modality (body part = forearm). Abbreviations 'Gabor', 'GLCM' and 'LBP' respectively stand for: Gabor ${ }_{4,6}^{r i}, \mathrm{GLCM}_{1}^{r i}+\mathrm{GLCM}_{2}^{r i}$ and $\mathrm{LBP}_{8,1}^{r i u 2}+\mathrm{LBP}_{16,2}^{r i u 2}$. The points on the diagonal report the accuracy attained by each method alone, the others the accuracy obtained by concatenating the two descriptors indicated in the the corresponding row and column.

\subsection{Classification}

Classification was based on three strategies: 1) nearest-neighbour (1-NN) rule with $L_{2}$ distance; 2) Support Vector Machines (SVMs) with linear kernels and 3) SVMs with radial basis kernels. Two-class SVMs were trained in a one-vs-all fashion and the maximum score was considered. Optimal values for $C$ and $\gamma$ were grid-searched in the domains $C \in\left\{2^{0}, 2^{2}, \ldots, 2^{8}\right\}$ and $\gamma=\left\{2^{-8}, 2^{-6}, \ldots, 2^{8}\right\}$ through 5-fold randomised split-half cross validation on the train set (see Sec. 4).

\section{Experiments}

To assess the effectiveness of the proposed method we carried out two groups of multi-class supervised classification experiments.

- In first group we evaluated the recognition accuracy obtained by applying the image descriptors - classifier pairs to either Epsilon or Proscope images from each of the the back of the hand, forearm or palm considered separately. No feature combination was used in this first group of tests.

- In the second we investigated various joint combinations of image features (i.e.: Gabor filters, GLCM and LBP) and of the two imaging modalities (i.e.: Epsilon and Proscope).

In all the experiments accuracy estimation was based on 100-fold cross-validation with stratified sampling. In each classification problem three samples of each class were used to train the classifier (train set) and the remaining one to test the accuracy (test set). The accuracy was estimated as the fraction of samples of the test set correctly classified, and the value was averaged over the 100 random subdivisions into train and test set.

\section{Results and discussion}

Table 2 and Fig. 6 summarize the results of the first group of experiments. The figures clearly show that skin texture features from the forearm yielded higher accuracy than those obtained from the skin of the back of the hand and of the palm. Among the image descriptors Gabor filters and LBP gave the best results and consistently outperformed GLCM. As the right column of Tab. 2 shows, images obtained with the Epsilon sensor yield a consistently good response. Recognition rates are generally better than those achieved with the optical sensor, which however attains the lowest error rates with the right choice of features.

The results from the second group of experiments (Tab. 3 and Fig. 7) show that the overall classification accuracy can be appreciably increased by combining the image descriptors and the two imaging modalities. Notably, capacitive imaging appears to provide a significant boost with respect to the optical modality alone - irrespectively of the skin area considered. As for the absolute accuracy values, we observe that classification based on rbk-SVM and a combination of Gabor and LBP features extracted from both dermoscopic and capacitive images of the inner forearm yielded close to $96 \%$ accuracy. It is also worth noting that over $94 \%$ accuracy was obtained with the same combination of features and a simple parameter-free classifier (1-NN), which testifies to the goodness of the features. Crucially, for all feature combinations and image modalities inner forearm images yielded better recognition performance than either palm images or the back of the hand. 


\section{Conclusions}

We investigated personal identification based on skin texture features from the inner forearm and a combination of optical and capacitive imaging. Importantly for practical reasons, the proposed approach requires neither registration of the optical and capacitive images, nor that they represent the same patch of skin between them or across different acquisitions. No particular care needs to be expended in positioning the sensors on the forearm in spite of the small imaging area.

The experiments showed that overall classification accuracy approaching $96 \%$ can be obtained by combining standard texture descriptors into an rbk-SVM classifier. Interestingly, forearm texture yielded significantly better results than were obtained by the same procedure when applied to the skin of the back of the hand and of the palm. This suggests that other authentication algorithms for palm texture or hand texture authentication might see their accuracy improved if they were applied to forearm skin. Conversely, we found that capacitive imaging consistently provided a significant performance boost with respect to the optical modality alone - regardless of the skin area inspected - and could therefore be considered as a complementary modality to multispectral approaches.

In conclusion, forearm skin texture appears to be a relevant source of information for biometric identification. The relatively easy accessibility of this area, together with the fact that it is normally shielded from the elements and wear to which other parts are exposed, make it a viable and convenient biometric modality in cases where multimodal authentication is required to achieve high security or when fingerprints cannot be used.

\section{Limitations and future work}

The present study is eminently cross-sectional, so no attempt was made to evaluate the intra-class variability of forearm skin texture over time. It seems however safe at least to assume that the potential changes undergone by skin texture from the forearm should be comparable with those affecting other skin areas (e.g. fingertips, palm, back of the hand, etc.) that represent commonly accepted biometrics - and which are considered fairly stable in the short and medium term [16]. Further studies might nevertheless be required to validate this assumption experimentally. Another interesting direction for future research could be the evaluation of different strategies for combining the various image features and imaging modalities.

\section{Acknowledgements}

F. Bianconi wishes to acknowledge the support offered by the Department of Engineering at the Università degli Studi di Perugia, Italy, under projects '41IMMA13FB' and 'BioMeTron - Fundamental research - D.D. 20/2015'; P. Xiao thanks London South Bank University for the financial support and Biox Systems Ltd for the studentship of C. Bontozoglou.

[1] N.A. Vashi, M.B. De Castro Maymone, and R.V. Kundu. Aging differences in ethnic skin. Journal of Clinical and Aesthetic Dermatology, 9(1):31-38, 2016

[2] M. Turk and A. Pentland. Eigenfaces for recognition. Journal Cognitive Neuroscience (Winter), 3(1):71-86, 1991.

[3] B. Duc, S. Fischer, and J. Bigün. Face authentication with Gabor information on deformable graphs. IEEE Transactions on Image Processing, 8(4):504-516, 1999.

[4] A. Tefas, C. Kotropoulos, and I. Pitas. Using support vector machines to enhance the performance of elastic graph matching for frontal face authentication. IEEE Transactions on Pattern Analysis and Machine Intelligence, 23(7):735-746, 2001.

[5] M. Bicego, A. Lagorio, E. Grosso, and M. Tistarelli. On the use of SIFT features for face authentication. In Proceedings of the Computer Vision and Pattern Recognition Workshop (CVPRW'06), pages 35-35, New York, NY, USA, 62006

[6] W.-Y. Han and J.-C. Lee. Palm vein recognition using adaptive Gabor filter. Expert Systems with Applications, 39(18):13225-13234, 2012.

[7] Office for National Statistics. Harmonised concepts and questions for social data sources. primary principles. version 3.3, 2015. Available online http://www.ons.gov.uk/ons/guide-method/harmonisation/ primary-set-of-harmonised-concepts-and-questions/ethnic-group.pdf. Last accessed on November $26,2015$.

[8] X. Ou, W. Pan, and P. Xiao. In vivo skin capacitive imaging analysis by using grey level co-occurrence matrix (GLCM). International Journal of Pharmaceutics, 460(1-2):28-32, 2014.

[9] W. Pan, X. Zhang, E. Chirikhina, and P. Xiao. In-vivo skin imaging for hydration and micro relief measurements. In Proccesings of Stratum Corneum V conference, Cardiff, UK, 72007.

[10] Bodelin Technologies. Proscope HR specifications, 2015. Available online https://www.bodelin.com/files/proscope/docs/ ProScopeHR2_Specifications.pdf. Last accessed on November 26, 2015.

[11] F. Bianconi and A. Fernández. Evaluation of the effects of Gabor filter parameters on texture classification. Pattern Recognition, 40(12):33253335, 2007. 
[12] F. Lahajnar and S. Kovacic. Rotation-invariant texture classification. Pattern Recognition Letters, 24(9-10):1151-1161, 2003.

[13] R. M. Haralick, K. Shanmugam, and I. Dinstein. Textural features for image classification. IEEE Transactions on Systems, Man, and Cybernetics, 3(6):610-621, 1973.

[14] F. Bianconi and A. Fernández. Rotation invariant co-occurrence features based on digital circles and discrete Fourier transform. Pattern Recognition Letters, 48:34-41, 2014.

[15] T. Ojala, M. Pietikäinen, and T. Mäenpää. Multiresolution gray-scale and rotation invariant texture classification with local binary patterns. IEEE Transactions on Pattern Analysis and Machine Intelligence, 24(7):971-987, 2002.

[16] J. Xie, L. Zhang, J. You, D. Zhang, and X. Qu. A study of hand back skin texture patterns for personal identification and gender classification. Sensors, 12(7):8691-8709, 2012. 
Table 1: Image descriptors: round-up table.

\begin{tabular}{ccc}
\hline Image descriptor & Acronyms and variations & No. of features \\
\hline \multirow{2}{*}{ Grey-level co-occurrence matrices } & $\mathrm{GLCM}_{1}^{r i}$ & 20 \\
& $\mathrm{GLCM}_{2}^{r i}$ & 50 \\
\hline Gabor filters & $\mathrm{Gabor}_{4,6}^{r i}$ & 48 \\
\hline \multirow{2}{*}{ Local Binary Patterns } & $\mathrm{LBP}_{8,1}^{\text {riu }}$ & 10 \\
& $\mathrm{LBP}_{16,2}^{\text {riu2 }}$ & 18 \\
\hline
\end{tabular}

Table 2: Overall recognition accuracy (in \%) by image descriptor, image source, acquisition zone and classifier. The random baseline accuracy for 43 subjects is $2.32 \%$

\begin{tabular}{|c|c|c|c|c|c|c|c|c|c|c|}
\hline \multirow{2}{*}{ Classifier } & \multirow{2}{*}{ Image descriptor } & \multicolumn{3}{|c|}{ Epsilon } & \multicolumn{3}{|c|}{ Proscope } & \multicolumn{3}{|c|}{ diff. (Epsilon - Proscope) } \\
\hline & & $\mathrm{BH}$ & FR & PL & $\mathrm{BH}$ & FR & PL & $\mathrm{BH}$ & FR & PL \\
\hline \multirow{3}{*}{$1-\mathrm{NN}\left(L_{2}\right)$} & Gabor $_{4,6}^{r i}$ & 50.79 & 70.19 & 47.72 & 33.02 & 76.28 & 36.07 & 17.77 & -6.09 & 11.65 \\
\hline & $\mathrm{GLCM}_{1}^{r i}+\mathrm{GLCM}_{2}^{r i}$ & 27.30 & 49.33 & 28.44 & 21.84 & 48.26 & 18.09 & 5.46 & 1.07 & 10.35 \\
\hline & $\mathrm{LBP}_{8,1}^{r i u 2^{1}}+\mathrm{LBP}_{16,2}^{r i u 2^{2}}$ & 52.05 & 69.56 & 42.16 & 38.28 & 64.30 & 35.21 & 13.77 & 5.26 & 6.95 \\
\hline \multirow{3}{*}{ Linear-SVM } & Gabor $_{4,6}^{r i}$ & 57.26 & 73.23 & 48.88 & 38.30 & 78.12 & 42.14 & 18.96 & -4.89 & 6.74 \\
\hline & $\mathrm{GLCM}_{1}^{4,0}+\mathrm{GLCM}_{2}^{r i}$ & 27.67 & 43.63 & 26.33 & 19.84 & 41.33 & 22.44 & 7.83 & 2.30 & 3.89 \\
\hline & $\mathrm{LBP}_{8,1}^{r i u 2^{1}}+\mathrm{LBP}_{16,2}^{r i u 2^{2}}$ & 41.47 & 63.37 & 37.91 & 32.58 & 49.30 & 30.35 & 8.89 & 14.07 & 7.56 \\
\hline \multirow{3}{*}{ Rbk-SVM } & Gabor $_{4,6}^{r i}$ & 56.40 & 72.42 & 49.95 & 37.77 & 79.26 & 42.79 & 18.63 & -6.84 & 7.16 \\
\hline & $\mathrm{GLCM}_{1}^{r i}+\mathrm{GLCM}_{2}^{r i}$ & 40.77 & 59.42 & 36.88 & 31.93 & 57.00 & 27.47 & 8.84 & 2.42 & 9.41 \\
\hline & $\operatorname{LBP}_{8,1}^{r i u 2}+\operatorname{LBP}_{16,2}^{r i u 2}$ & 62.00 & 76.16 & 52.51 & 46.72 & 73.56 & 46.49 & 15.28 & 2.60 & 6.02 \\
\hline
\end{tabular}

$\mathrm{BH}, \mathrm{FR}$ and PL respectively indicate the back of the hand, forearm and palm.

Table 3: Overall recognition accuracy (in \%) obtained by combining image descriptors and imaging modalities.

\begin{tabular}{|c|c|c|c|c|c|c|c|c|c|c|}
\hline \multirow{2}{*}{ Classifier } & \multirow{2}{*}{ Image descriptor } & \multicolumn{3}{|c|}{ Epsilon } & \multicolumn{3}{|c|}{ Proscope } & \multicolumn{3}{|c|}{ Epsilon + Proscope } \\
\hline & & $\mathrm{BH}$ & FR & PL & $\mathrm{BH}$ & FR & PL & $\mathrm{BH}$ & FR & PL \\
\hline \multirow{3}{*}{$1-\mathrm{NN}\left(L_{2}\right)$} & \multirow{3}{*}{$\begin{array}{l}\text { Gabor }_{4,6}^{r i}+\mathrm{LBP}_{8,1}^{r i u 2}+\mathrm{LBP}_{16,2}^{r i u 2} \\
\operatorname{Gabor}_{4,6}^{r i}+\mathrm{GLCM}_{1}^{r i}+\mathrm{GLCM}_{2}^{r i} \\
\mathrm{LBP}_{8,1}^{r i u 2}+\mathrm{LBP}_{16,2}^{r i u 2}+ \\
\mathrm{GLCM}_{1}^{r i}+\mathrm{GLCM}_{2}^{r i}\end{array}$} & 63.56 & 87.16 & 60.98 & 38.47 & 78.72 & 38.30 & 71.81 & 94.30 & 65.91 \\
\hline & & 51.30 & 69.14 & 59.07 & 36.05 & 75.56 & 38.05 & 66.49 & 93.23 & 67.65 \\
\hline & & 55.93 & 71.98 & 49.05 & 36.67 & 66.05 & 35.51 & 54.35 & 81.02 & 52.77 \\
\hline \multirow{3}{*}{ Linear-SVM } & \multirow{3}{*}{$\begin{array}{l}\text { Gabor }_{4,6}^{r i}+\mathrm{LBP}_{8,1}^{r i u 2}+\mathrm{LBP}_{16,2}^{r i u 2} \\
\text { Gabor }_{4,6}^{r i}+\mathrm{GLCM}_{1}^{r i}+\mathrm{GLCM}_{2}^{r i} \\
\mathrm{LBP}_{8,1}^{r i u 2}+\mathrm{LBP}_{16,2}^{r i u 2}+ \\
\mathrm{GLCM}_{1}^{r i}+\mathrm{GLCM}_{2}^{r i}\end{array}$} & 55.81 & 74.67 & 55.12 & 39.84 & 72.51 & 40.95 & 74.93 & 93.79 & 72.42 \\
\hline & & 49.58 & 65.02 & 50.56 & 34.12 & 71.26 & 36.95 & 65.12 & 92.88 & 68.67 \\
\hline & & 42.93 & 67.35 & 40.95 & 33.72 & 48.86 & 32.07 & 56.53 & 80.77 & 53.23 \\
\hline \multirow{3}{*}{ Rbk-SVM } & \multirow{3}{*}{$\begin{array}{l}\mathrm{Gabor}_{4,6}^{r i}+\mathrm{LBP}_{8,1}^{r i u 2}+\mathrm{LBP}_{16,2}^{r i u 2} \\
\text { Gabor }_{4,6}^{r i}+\mathrm{GLCM}_{1}^{r i}+\mathrm{GLCM}_{2}^{r i} \\
\mathrm{LBP}_{8,1}^{r i u 2}+\mathrm{LBP}_{16,2}^{r i u 2}+ \\
\mathrm{GLCM}_{1}^{r i}+\mathrm{GLCM}_{2}^{r i}\end{array}$} & 70.53 & 87.07 & 64.26 & 48.81 & 82.44 & 52.98 & 77.67 & 95.77 & 75.26 \\
\hline & & 63.49 & 74.93 & 61.63 & 40.40 & 80.53 & 44.12 & 72.47 & 94.74 & 73.51 \\
\hline & & 67.60 & 75.30 & 55.86 & 47.77 & 74.09 & 48.26 & 67.74 & 85.21 & 63.86 \\
\hline
\end{tabular}

